\section{Mobilität, elektrophoretische}

R. Westermeier

Freising, Deutschland

Synonym(e) Elektrophoretische Beweglichkeit; Wanderungsgeschwindigkeit, elektrophoretische

Englischer Begriff electrophoretic mobility

Definition Die elektrophoretische Mobilität ist eine substanzspezifische Größe, welche die Wanderungsgeschwindigkeit eines Partikels im elektrischen Feld und über diese die Wanderungsstrecke eines Analyten von der Probenaufgabestelle innerhalb einer definierten Elektrophoresezeit bestimmt. Sie ist für die Qualität der elektrophoretischen Trennung von entscheidender Bedeutung.

Beschreibung Substanzen mit unterschiedlichen elektrophoretischen Mobilitäten können mit der $>$ Elektrophorese aufgetrennt werden. Die elektrophoretische Mobilität ist eine charakteristische physikochemische Eigenschaft eines Moleküls und abhängig von $>$ Nettoladung und Größe des Moleküls in einem vorgegebenen $\mathrm{pH}-\mathrm{Milieu}$.
Amphotere Moleküle, wie z. B. Proteine, haben bei einem $\mathrm{pH}$-Wert, der ihrem isoelektrischen Punkt entspricht, eine elektrophoretische Mobilität von Null. Sie würden also im elektrischen Feld nicht wandern. Je weiter ein $>$ isoelektrischer Punkt eines amphoteren Moleküls vom pH-Milieu entfernt ist, umso höher sind die Nettoladung und damit auch die elektrophoretische Mobilität. Lipide und andere nichtpolare Substanzen besitzen keine elektrophoretische Mobilität, da sie keine Nettoladung haben. Nukleinsäuren besitzen nur im basischen und neutralen, nicht aber sauren Milieu eine elektrophoretische Mobilität, weil sie nur dann negativ geladen sind.

Die Wanderungsgeschwindigkeit eines Moleküls im elektrischen Feld ist abhängig von seiner elektrophoretischen Mobilität (Beweglichkeit), der Siebwirkung oder Viskosität des Mediums, der Temperatur, der Ionenstärke des Puffers und der elektrischen Feldstärke.

\section{Literatur}

Lottspeich F, Engels JW (Hrsg) (2012) Bioanalytik, 3. Aufl. Spektrum Akademischer Verlag, Heidelberg

Westermeier R (2016) Elektrophorese leicht gemacht. VCH, Weinheim 